\title{
Fluorescence errors in integrating sphere measurements of remote phosphor type LED light sources
}

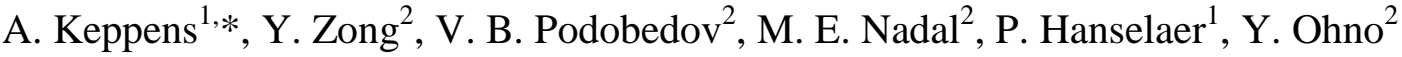 \\ ${ }^{1}$ Light \& Lighting Laboratory, Catholic University College Gent, Belgium, and ESAT/ELECTA, \\ K.U.Leuven, Belgium. \\ ${ }^{2}$ Optical Technology Division, National Institute of Standards and Technology (NIST), \\ Gaithersburg, MD, USA. \\ * This work has been performed while working as a Guest Researcher at NIST.
}

\begin{abstract}
The relative spectral radiant flux error caused by phosphor fluorescence during integrating sphere measurements is investigated both theoretically and experimentally. Integrating sphere and goniophotometer measurements are compared and used for model validation, while a case study provides additional clarification. Criteria for reducing fluorescence errors to a degree of negligibility as well as a fluorescence error correction method based on simple matrix algebra are presented. Only remote phosphor type LED light sources are studied because of their large phosphor surfaces and high application potential in general lighting.
\end{abstract}

Keywords: integrating sphere, spectral radiant flux, fluorescence error, remote phosphor type LED light source

\section{INTRODUCTION}

Integrating spheres (Ulbricht spheres) are the preferred instruments for determining light sources' (spectral) radiant or luminous flux, both at industrial and academic measurement facilities ${ }^{[1][2]}$. Main reason is the instantaneous spatial integration compared to time consuming goniophotometer measurements. However, several industrial sources reported significant luminous flux errors in integrating sphere measurements caused by fluorescence of phosphor type LED lamps. This error is typically observed in self-absorption measurements for relatively small integrating spheres, and is due to fluorescence of the phosphor excited by the emission from the calibration or auxiliary lamp. The self-absorption is not determined correctly as a result. A similar error occurs in the flux measurement of the phosphor type LED lamp due to excitation of the phosphor by the reflected radiant flux in the sphere. In both cases, the measured spectrum shows a lower blue component and a higher yellow component than the true flux spectrum. This relative spectral radiant flux error depends on the spectral power distribution of the light source and thus on the source type under test, e.g. calibration source, auxiliary source, or LED source. Therefore, straightforward calibration methods do not annihilate this effect and supplementary corrections have to be considered ${ }^{[3][4]}$.

This work investigates the relative spectral radiant flux error caused by phosphor fluorescence during integrating sphere measurements both theoretically and experimentally. Phosphors' spectral radiance coefficient and 
spectral fluorescence coefficient are first determined by use of a double monochromator and goniophotometer. Radiant fluxes of the LED light sources are measured with an ambient-temperature-controlled integrating sphere afterwards. Phosphor excitation with an external blue diode allows evaluating the fluorescence contribution due to self-absorption during sphere measurements separately. Integrating sphere and goniophotometer measurements are compared and used for model validation, while a case study provides additional clarification. The final goal of this work is to propose an addendum to existing standards for flux measurements of phosphor-white LEDs with integrating spheres. Criteria for reducing fluorescence errors to a degree of negligibility as well as a fluorescence error correction method based on simple matrix algebra are presented. Only remote phosphor type LED light sources are studied because of their large phosphor surfaces and high application potential in general lighting ${ }^{[5]}$.

\section{THEORY}

Using spherical coordinates in an integrating sphere with radius $R$, the spectral irradiance $E_{e, \lambda} \lambda$ at a position $\alpha, \beta$ is given by

$$
E_{e, \lambda} \lambda, \alpha, \beta=\frac{1}{4 R^{2}} \int L_{e, \lambda} \lambda, \alpha, \beta, \theta, \varphi d A
$$

with $L_{e, \lambda} \lambda, \alpha, \beta, \theta, \varphi$ the spectral radiance at point $\theta, \varphi$ in the direction of $\alpha, \beta$ and the surface integral covering all points $\theta, \varphi$ within the integrating sphere that can be seen from $\alpha, \beta$ directly ${ }^{[4][6]}$.

The following premises are taken into account:

(a) For merely reflecting surfaces, the spectral radiance at each point can be written as a function of the spectral irradiance as follows ${ }^{[7]}$ :

$$
L_{e, \lambda} \lambda, \alpha, \beta, \theta, \varphi=q_{e} \lambda, \alpha, \beta E_{e, \lambda} \lambda, \theta, \varphi
$$

with $q_{e} \lambda, \alpha, \beta$ the spectral radiance coefficient for a diffuse irradiation geometry in the direction $\alpha, \beta$. This coefficient is independent of the direction of view for Lambertian surfaces with overall spectral reflectance $\rho \lambda$ :

$$
L_{e, \lambda} \lambda, \alpha, \beta, \theta, \varphi=\frac{\rho \lambda}{\pi} E_{e, \lambda} \lambda, \theta, \varphi
$$

(b) Spectral radiances of surfaces with different radiance coefficients have to be considered separately. Coefficients $q_{e, s}, q_{e, h}$, and $q_{e, p}$ are attributed to the integrating sphere inner surface or baffles, light source housing and mounting, and remote phosphor, respectively.

(c) The spectral radiance of the remote phosphor caused by fluorescence after self-absorption can be written as

$$
L_{e, \lambda} \lambda, \alpha, \beta, \theta, \varphi=\int f_{\lambda} \lambda, \lambda^{\prime} E_{e, \lambda} \lambda^{\prime}, \theta, \varphi d \lambda^{\prime}
$$

with $f_{\lambda} \lambda, \lambda^{\prime}$ the spectral fluorescence coefficient (also called spectral fluorescence quantum yield) and the integral covering all (visible) wavelengths $\lambda^{\prime}<\lambda^{[8]}$. The spectral fluorescence coefficient is defined as the radiance caused by fluorescence at wavelength $\lambda$ proportional to the irradiance at wavelength $\lambda^{\prime}$ and is considered independent of the direction of view. The total spectral radiance $L_{e, \lambda} \lambda$ emerging from a point $\theta, \varphi$ on the phosphor surface in the direction $\alpha, \beta$ thus equals:

$$
L_{e, \lambda} \lambda, \alpha, \beta, \theta, \varphi=q_{e, p} \lambda, \alpha, \beta E_{e, \lambda} \lambda, \theta, \varphi+\int f_{\lambda} \lambda, \lambda^{\prime} E_{e, \lambda} \lambda^{\prime}, \theta, \varphi d \lambda^{\prime}
$$


(d) Deviations from the ideal integrating sphere configuration due to baffles, wall openings, or light source mounting are considered to be small, so that the $1 / 4 R^{2}$ fore factor in Eq. (1) can be maintained as an approximation.

When a calibration lamp, auxiliary lamp, or remote phosphor type LED lamp is mounted inside the integrating sphere, it must always be shielded from the detector by a baffle. Calibration sources are sometimes mounted outside the sphere in front of a sphere wall opening ${ }^{[4]}$. In both cases, when one of the lamps is switched on, the spectral irradiance at the receiver's position $\alpha_{r}, \beta_{r}$ becomes

$$
E_{e, \lambda} \lambda, \alpha_{r}, \beta_{r}=\frac{1}{4 R^{2}} \int_{s}^{\rho_{s} \lambda} \frac{\lambda}{\pi} E_{e, \lambda} \lambda, \theta, \varphi d A_{s}
$$

by combining Eqs. (1) and (3), and in the assumption of a Lambertian response for the inner integrating sphere coating. $s$ denotes the sphere wall (or baffle) surface that can be seen by the receiver directly. Eq. (6) can be rewritten as

$$
E_{e, \lambda} \lambda, \alpha_{r}, \beta_{r}=\frac{\rho_{s} \lambda}{4 \pi R^{2}} \Phi_{e, \lambda}^{\Sigma} \lambda
$$

if $\Phi_{e, \lambda}^{\Sigma} \lambda$ represents the total spectral radiant flux inside the integrating sphere during radiative equilibrium. Calculation of the spectral irradiance at the receiver's position thus requires an expression for the total spectral radiant flux inside the sphere. $\Phi_{e, \lambda}^{\Sigma} \lambda$ equals the sum of the initial spectral radiant flux emitted by the light source $\Phi_{e, \lambda}^{0} \lambda$ and subsequent interactions of this initial flux with all elements within the integrating sphere. These subsequent interactions can be taken into account by expressing the spectral irradiance after $n+1$ interactions at a random point $\alpha, \beta$ as a function of the preceding spectral irradiances $n$ at all other points:

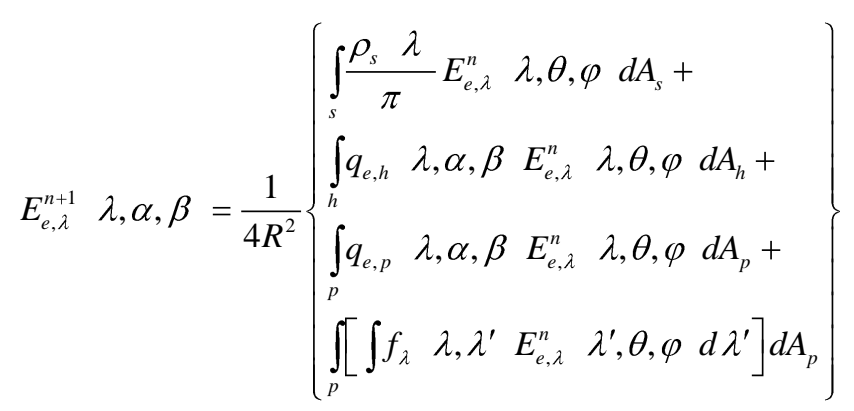

through combination of Eq. (1) with Eqs. (2) and (5), applied to the corresponding surface materials. $h$ and $p$ denote the light source housing and mounting surface and remote phosphor surface, respectively.

The first three terms in Eq. (8) can be rewritten as an averaged spectral irradiance, weighed by the respective surface ratios. Denoting the housing to sphere surface ratio and phosphor to sphere surface ratio with $R_{h}$ and $R_{p}$ respectively, one obtains

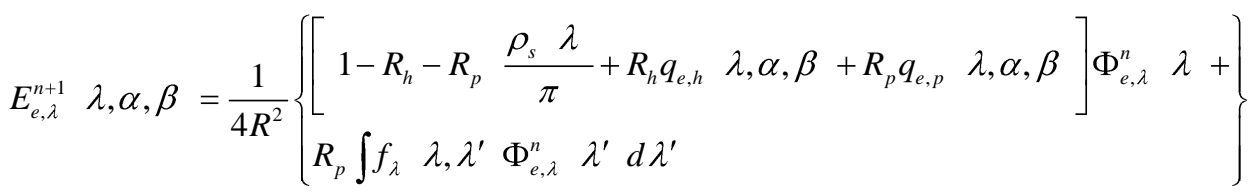

$$
\begin{aligned}
& =\frac{1}{4 R^{2}}\left[\bar{q}_{e} \lambda, \alpha, \beta \Phi_{e, \lambda}^{n} \lambda+R_{p} \int f_{\lambda} \lambda, \lambda^{\prime} \Phi_{e, \lambda}^{n} \lambda^{\prime} d \lambda^{\prime}\right]
\end{aligned}
$$


with $\Phi_{e, \lambda}^{n} \lambda$ the spectral radiant flux contribution from interaction $n$ of the initial flux with all elements within the sphere. Factor $\bar{q}_{e} \lambda, \alpha, \beta$ equals the first term between brackets and corresponds to the average spectral radiance coefficient.

Integration of both sides of Eq. (9) over all points on the inner sphere surface yields

$$
\Phi_{e, \lambda}^{n+1} \lambda=\pi \bar{q}_{e} \lambda \Phi_{e, \lambda}^{n} \lambda+\pi R_{p} \int f_{\lambda} \lambda, \lambda^{\prime} \Phi_{e, \lambda}^{n} \lambda^{\prime} d \lambda^{\prime}
$$

if deviations from the ideal sphere configuration due to baffles, wall openings, or light source mounting are again neglected (see premise (d)). Note that the integration also annihilates the directionality of the averaged spectral radiance coefficient. As a result, $\pi \bar{q}_{e} \lambda$ may be replaced by the averaged sphere reflectance $\bar{\rho} \lambda$. For $f_{\lambda} \lambda, \lambda^{\prime}$ equal to zero for all wavelengths, Eq. (10) thus agrees with regular integrating sphere theory.

The total spectral radiant flux inside the integrating sphere can now be calculated as follows:

$$
\begin{aligned}
& \Phi_{e, \lambda}^{\Sigma} \lambda=\sum_{n=0}^{\infty} \Phi_{e, \lambda}^{n} \lambda \\
& =\Phi_{e, \lambda}^{0} \lambda+\Phi_{e, \lambda}^{1} \lambda+\Phi_{e, \lambda}^{2} \lambda+\cdots \\
& =\Phi_{e, \lambda}^{0} \lambda \\
& +\left[\begin{array}{lllllll}
\bar{\rho} \lambda & \Phi_{e, \lambda}^{0} & \lambda+\pi R_{p} \int f_{\lambda} & \lambda, \lambda^{\prime} & \Phi_{e, \lambda}^{0} & \lambda^{\prime} & d \lambda^{\prime}
\end{array}\right]
\end{aligned}
$$

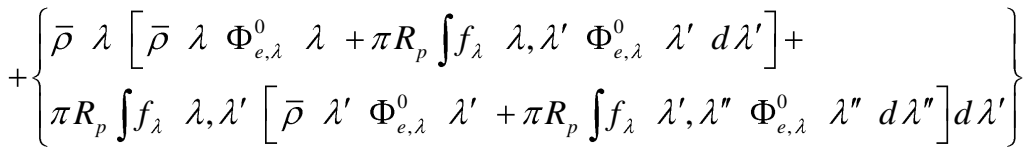

$$
\begin{aligned}
& +\cdots
\end{aligned}
$$

The infinite sum can be simplified by taking into account that the light emitted by the phosphor through fluorescence cannot be absorbed for re-emission by that same phosphor $\left(\lambda^{\prime}>\lambda\right)$. Terms containing successive fluorescence contributions will therefore not occur, yielding:

$$
\begin{aligned}
& \Phi_{e, \lambda}^{\Sigma} \lambda=\Phi_{e, \lambda}^{0} \lambda \\
& +\left[\begin{array}{lllllll}
\bar{\rho} & \lambda & \Phi_{e, \lambda}^{0} & \lambda+\pi R_{p} \int f_{\lambda} \lambda, \lambda^{\prime} & \Phi_{e, \lambda}^{0} & \lambda^{\prime} & d \lambda^{\prime}
\end{array}\right]
\end{aligned}
$$

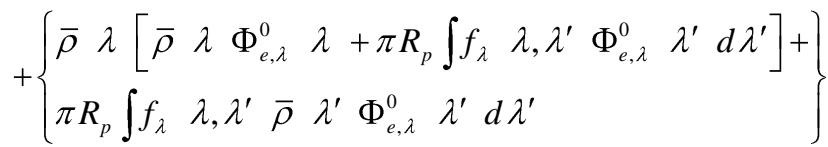

$$
\begin{aligned}
& +\cdots \\
& =\left[\begin{array}{cc}
1+\bar{\rho} & \lambda+\bar{\rho}^{2} \lambda+\cdots
\end{array}\right] \Phi_{e, \lambda}^{0} \lambda
\end{aligned}
$$

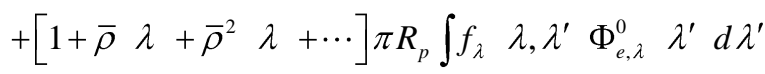

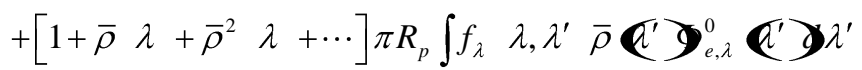

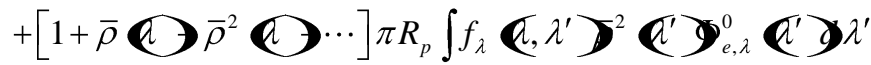

$$
\begin{aligned}
& +\cdots \\
& =\left[\frac{1}{1-\bar{\rho}(2)}\right]\left\{\Phi_{e, \lambda}^{0} @ \pi R_{p} \int f_{\lambda}\left(\lambda^{\prime} \lambda^{\prime}\left[\frac{1}{1-\bar{\rho} \bar{l}^{\prime}}\right] \Phi_{e, \lambda}^{0} \mathbb{e}^{\prime} \partial \lambda^{\prime}\right\}\right.
\end{aligned}
$$


This final result makes sense. In the absence of fluorescence due to self-absorption, the total spectral radiant flux inside

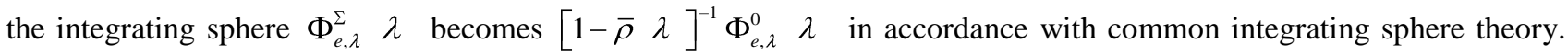
The last term in Eq. (12) represents the fluorescence contribution of the phosphor due to partial self-absorption of the

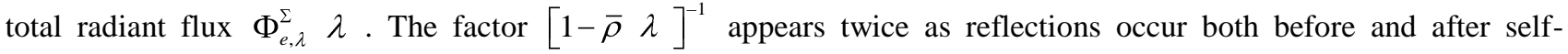
absorption.

Combining Eqs. (1) and (12), the spectral irradiance at the receiver's position becomes:

$$
E_{e, \lambda} \lambda, \alpha_{r}, \beta_{r}=\frac{1}{4 \pi R^{2}}\left[\frac{\rho_{s} \lambda}{1-\bar{\rho} \lambda}\right]\left[\begin{array}{llll}
\Phi_{e, \lambda}^{0} \lambda+\pi R_{p} \frac{f_{\lambda} \lambda, \lambda^{\prime}}{1-\bar{\rho} \lambda^{\prime}} \Phi_{e, \lambda}^{0} & \left.\lambda^{\prime} d \lambda^{\prime}\right]
\end{array}\right]
$$

The spectral relative error $\varepsilon_{e, r, \lambda} \lambda$ caused by the fluorescence contribution to the receiver's spectral irradiance can be calculated as the quotient of the second and first term in Eq. (13):

$$
\varepsilon_{e, r, \lambda} \lambda=\frac{\pi R_{p}}{\Phi_{e, \lambda}^{0} \lambda} \int \frac{f_{\lambda} \lambda, \lambda^{\prime}}{1-\bar{\rho} \lambda^{\prime}} \Phi_{e, \lambda}^{0} \lambda^{\prime} d \lambda^{\prime}
$$

As could be expected, the relative error depends on the initial spectral radiant flux of the source under test, the average reflectance of all elements within the integrating sphere, the phosphor to sphere surface ratio, and the phosphor's spectral fluorescence coefficient.

At first sight, it is remarkable that the blue radiant flux decrease is only implicitly incorporated into the relative error through the averaged spectral reflectance. This could however be expected, as the phosphor's spectral radiance coefficient $q_{e, p} \lambda$ corresponds with a spectrally non-uniform factor that is - as all terms in $\bar{\rho} \lambda$ - corrected for through calibration.

Analogous to Eq. (14), the total relative fluorescence error on luminous flux measurements with integrating spheres can be determined as

$$
\varepsilon_{r}=\frac{\int\left[\pi R_{p} \int \frac{f_{\lambda} \lambda, \lambda^{\prime}}{1-\bar{\rho} \lambda^{\prime}} \Phi_{e, \lambda}^{0} \lambda^{\prime} d \lambda^{\prime}\right] V \lambda d \lambda}{\int \Phi_{e, \lambda}^{0} \lambda V \lambda d \lambda}
$$

with $V \lambda$ the eye sensitivity function and the integrals covering all visible wavelengths $\lambda$ (380 $\mathrm{nm}$ to $780 \mathrm{~nm})^{[1]}$.

\section{EXPERIMENTS}

Two commercial remote phosphor type LED light sources are considered. The first (L1) contains a planar phosphor plate, the second (L2) a bulb-shaped phosphor cover. The phosphor surfaces amount $2.82710^{-3} \mathrm{~m}^{2}$ and $5.56210^{-3} \mathrm{~m}^{2}$, respectively. Each phosphor plate's spectral radiance coefficient and spectral fluorescence coefficient is first determined by use of a double monochromator and goniophotometer. Radiant fluxes of the LED light sources are measured with an ambient-temperature-controlled integrating sphere afterwards. Phosphor excitation with an external blue diode allows evaluating the fluorescence contribution due to self-absorption during sphere measurements separately. 


\subsection{Goniophotometer measurements}

Each phosphor plate's spectral radiance coefficient and spectral fluorescence coefficient is determined by use of a double monochromator and goniophotometer, combined into one measurement setup ${ }^{[9]}$. The double monochromator allows selecting a specific wavelength range (FWHM $\approx 5 \mathrm{~nm}$, per $10 \mathrm{~nm}$ ) from a continuous spectrum HID lamp. This incident beam hits the phosphor surface of the LED light source - positioned in the centre of the goniophotometer - under an angle $\theta_{i}$ with respect to the surface normal. Five incident beam angles are selected: $0^{\circ}, 20^{\circ}, 40^{\circ}, 60^{\circ}$, and $80^{\circ}$. For each incident wavelength and each incident beam angle $\theta_{i}$, the hemispherical phosphor response containing both radiance and fluorescence contributions is determined. Due to symmetry, response beams within only one sphere quadrant have to be measured with a spectrometer (see Fig. 1). The selected response beam directions $\theta_{r}, \varphi_{r}$ range from $10^{\circ}$ to $80^{\circ}$ per $10^{\circ}$ for $\theta_{r}$ and from $20^{\circ}$ to $180^{\circ}$ per $20^{\circ}$ for $\varphi_{r}$.

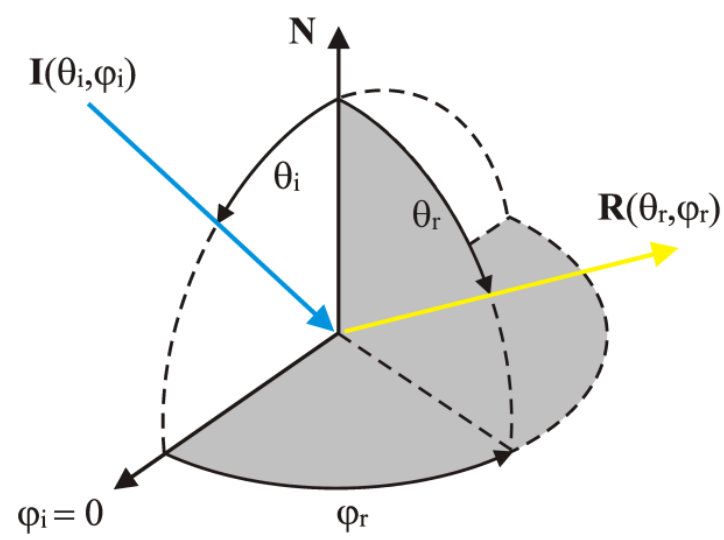

Fig. 1: Schematic representation of a phosphor response measurement. The incident beam $I$ with direction $\theta_{i}, \varphi_{i}=0$ respective to the phosphor surface normal $N$ causes reflections and fluorescence in a hemisphere. Response beams $R$ are spectrally measured in directions $\theta_{r}, \varphi_{r}$ of one sphere quadrant (grey area).

All spectral radiant intensity measurements of the phosphor response are first corrected for unwanted contributions of the spectrometer dark current, spectrometer stray light, and room dark current ${ }^{[10]}$. A spline interpolation technique is used to convert the data to $1 \mathrm{~nm}$ intervals. The spectral radiant intensities are then spatially integrated for each incident wavelength and each incident beam angle separately. A second spatial integration for all incident beam angles results in wavelength dependent radiance and fluorescence coefficients. Division of these quantities by their respective reference beams - with correction for source stability - allows calculating the spectral radiance coefficient (vector) and spectral fluorescence coefficient (matrix). Each phosphor's relative spectral response is determined as the sum of radiance and fluorescence contributions for a flat and unitary input spectrum (see Fig. 2). 

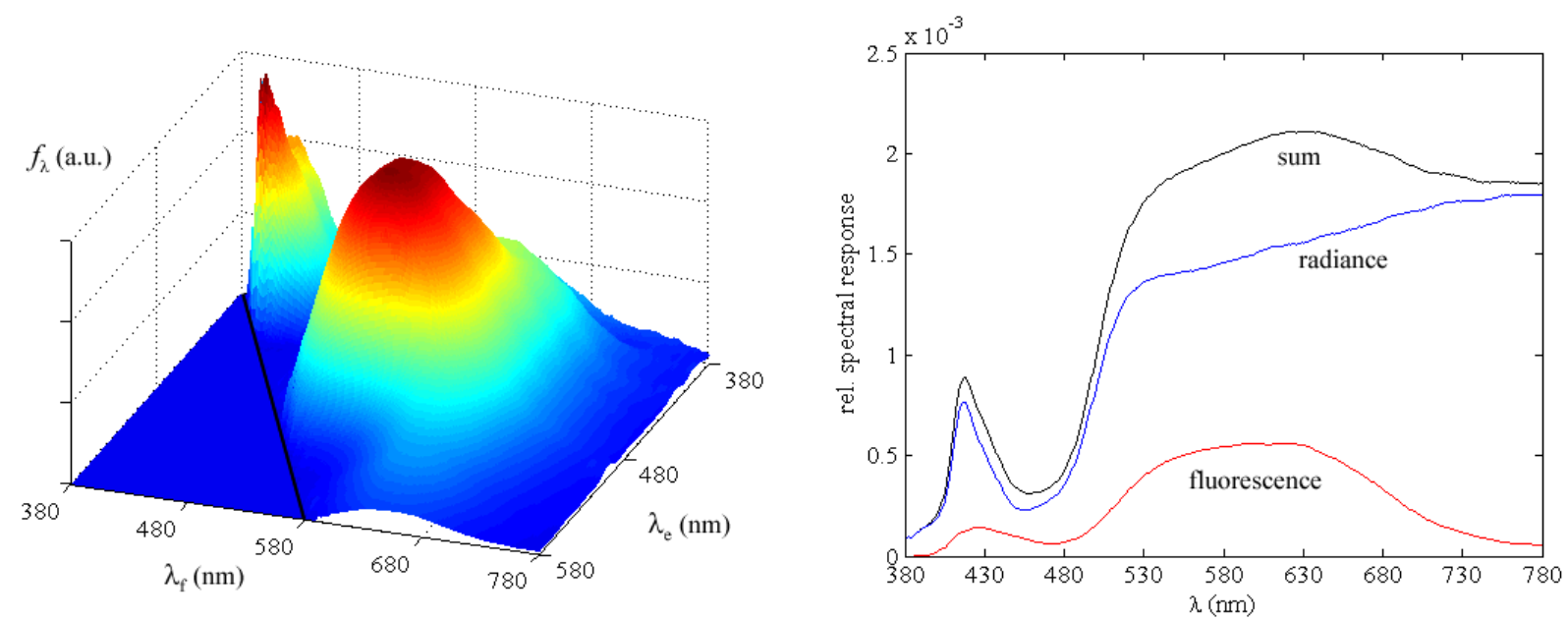

Fig. 2: Visualisation of the phosphor plate's spectral fluorescence coefficient for excitation wavelengths $\lambda_{e}$ and fluorescence wavelengths $\lambda_{f}$ (left) and relative spectral response (right). The blue line represents the spectral radiance contribution, the red line the spectral fluorescence contribution, and the black line equals their sum.

\subsection{Integrating sphere measurements}

Spectral radiant flux measurements with an ambient-temperature-controlled integrating sphere (one meter diameter) are performed for model validation. These include halogen auxiliary source measurements, LED light source measurements, and fluorescence measurements with an external blue LED for both sources under test L1 and L2. During each measurement, the phosphor temperature is kept constant at the same temperature as for the goniophotometer experiments, i.e. $295 \pm 1 \mathrm{~K}$. This temperature control is necessary due to the temperature dependence of phosphors' absorbance and external quantum efficiency ${ }^{[11][12]}$. The smaller effect of pump flux density on these quantities is neglected $^{[12]}$. For the external blue LEDs, a previously developed method is applied in order to keep the junction temperature and thus the radiant flux spectrum constant ${ }^{[13][14]}$.

As for all goniophotometer measurements, the integrating sphere spectra are corrected for unwanted contributions of the spectrometer dark current and stray light. Again a spline interpolation technique is used to convert the data to $1 \mathrm{~nm}$ intervals.

\section{RESULTS AND DISCUSSION}

\subsection{Model validation}

Measurement results are first utilized to validate Eq. (13) and its preceding derivation in section 2. Spectral radiant flux measurements of the external blue LED with and without the remote phosphor LED light sources mounted inside the sphere are compared. Adding the error term in Eq. (13) to the latter, i.e. the initial blue spectrum $\Phi_{e, \lambda}^{0} \lambda$, should result in the former (see Fig. 3). The spectral fluorescence coefficient $f_{\lambda} \lambda, \lambda^{\prime}$ is thereby obtained from goniophotometer measurements. The phosphor to sphere surface ratios $R_{p}$ equal $9.010^{-4}$ and $17.710^{-4}$ for L1 and L2, respectively, while the integrating sphere's average reflectance amounts 0.96 for all wavelengths by approximation. 


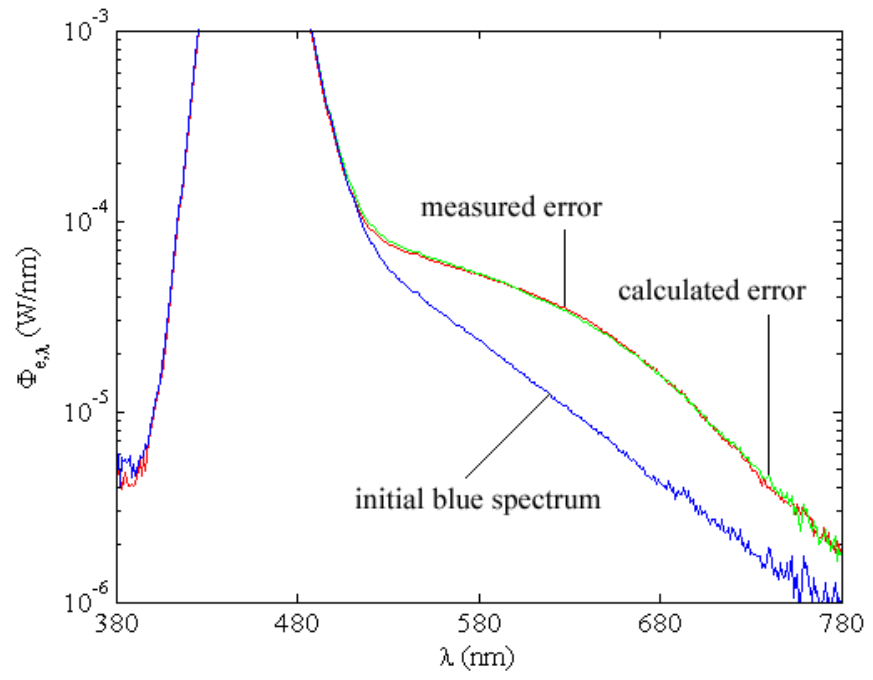

Fig. 3: Comparison of measured (red line) and calculated (green line) spectral radiant flux errors for L1, superposed to the initial blue spectrum (blue line) of an external LED source.

A very good agreement between measured and calculated spectral radiant flux errors has been found for both phosphor samples (see Fig. 3 for sample L1), with deviations smaller than $1 \%$. This confirms the theory that has been developed in the second section. Relative fluorescence errors on radiant fluxes $\varepsilon_{e, r}$ and on luminous fluxes $\varepsilon_{r}$ can thus be properly computed by use of Eqs. (14) and (15), respectively. Relative error values of both remote phosphor samples are gathered in Table 1. Similar errors are found in combination with the auxiliary source, when the remote phosphor LED light sources themselves are switched off.

Table 1: Relative fluorescence errors on radiant fluxes $\varepsilon_{e, r}$ and on luminous fluxes $\varepsilon_{r}$ for both remote phosphor samples and in combination with an auxiliary light source.

\begin{tabular}{|c|c|c|c|}
\hline light source & error source & $\varepsilon_{e, r}(\%)$ & $\varepsilon_{r}(\%)$ \\
\hline auxiliary & L1 & 0.17 & 0.25 \\
\hline L1 & L1 & 0.17 & 0.17 \\
\hline auxiliary & L2 & 2.83 & 3.24 \\
\hline L2 & L2 & 2.69 & 3.02 \\
\hline
\end{tabular}

The results in Table 1 learn that relative fluorescence errors can be of the order of a few percentages, making compensation efforts necessary. The error largely depends on the fluorescence coefficient of the remote phosphor under test. Fluorescence contributions are indeed expected to be significantly bigger for phosphors used for applications with low correlated colour temperature (CCT), related to high photon absorption coefficients. Samples L1 and L2 have CCTs of $4000 \mathrm{~K}$ and $3000 \mathrm{~K}$, respectively. Due to shape correspondences between fluorescence spectra and the $V \lambda$ eye sensitivity function, typically $\varepsilon_{r} \geq \varepsilon_{e, r}$.

\subsection{Case study}

Using Eq. (15), the total relative fluorescence error on luminous flux $\varepsilon_{r}$ is examined as a function of the average integrating sphere reflectance $\bar{\rho}$ and correlated colour temperature $T_{c}$ of a black body reference (or auxiliary) source 
(see Table 2). Because of its relatively high fluorescence error contributions (see Table 1), the phosphor bulb's fluorescence coefficient is taken as a reference. Surface ratio $R_{p}$ is set to $10^{-3}$, i.e. in between the phosphor to sphere surface ratios of the samples under consideration. As fluorescence errors linearly depend on the surface ratio, they can be easily recalculated for other $R_{p}$ values.

Table 2: Total relative fluorescence error on luminous flux (in \%) as a function of the average integrating sphere reflectance $\bar{\rho}$ (rows) and correlated colour temperature $T_{c}$ of a black body reference (or auxiliary) source (columns).

\begin{tabular}{|c|c|c|c|c|c|c|c|c|c|c|}
\hline $\bar{\rho} \backslash T_{c}$ & 2700 & 3000 & 3300 & 3600 & 3900 & 4200 & 4500 & 4800 & 5100 & 5400 \\
\hline 0.80 & 0.45 & 0.55 & 0.65 & 0.75 & 0.84 & 0.93 & 1.02 & 1.10 & 1.18 & 1.26 \\
\hline 0.82 & 0.50 & 0.61 & 0.72 & 0.83 & 0.93 & 1.04 & 1.13 & 1.23 & 1.32 & 1.40 \\
\hline 0.84 & 0.56 & 0.69 & 0.81 & 0.93 & 1.05 & 1.17 & 1.28 & 1.38 & 1.48 & 1.58 \\
\hline 0.86 & 0.64 & 0.78 & 0.93 & 1.07 & 1.20 & 1.33 & 1.46 & 1.58 & 1.69 & 1.80 \\
\hline 0.88 & 0.75 & 0.91 & 1.08 & 1.24 & 1.40 & 1.55 & 1.70 & 1.84 & 1.97 & 2.10 \\
\hline 0.90 & 0.90 & 1.10 & 1.30 & 1.49 & 1.68 & 1.86 & 2.04 & 2.21 & 2.37 & 2.52 \\
\hline 0.92 & 1.12 & 1.37 & 1.62 & 1.86 & 2.10 & 2.33 & 2.55 & 2.76 & 2.96 & 3.15 \\
\hline 0.94 & 1.50 & 1.83 & 2.16 & 2.49 & 2.80 & 3.11 & 3.40 & 3.68 & 3.95 & 4.20 \\
\hline 0.96 & 2.25 & 2.74 & 3.24 & 3.73 & 4.20 & 4.66 & 5.10 & 5.52 & 5.92 & 6.30 \\
\hline 0.98 & 4.49 & 5.49 & 6.48 & 7.46 & 8.40 & 9.32 & 10.20 & 11.04 & 11.85 & 12.61 \\
\hline
\end{tabular}

The results in Table 2 show that the relative fluorescence error increases with increasing average sphere reflectance and increasing CCT of the black body source. The effect of the former however is of major importance. Fluorescence errors increase by a factor ten for sphere reflectances going from 0.80 to 0.98 . A factor two difference occurs between the two highest reflectance coefficients. For sample L2, fluorescence errors obtained at high reflectances and CCTs are of the order of $10 \%$ and therefore unacceptable.

\subsection{Possible solutions}

It should be clear that fluorescence errors do not occur for goniophotometer or partial flux measurements ${ }^{[15]}$. Both approaches however lose the significant benefits of quick total (spectral) radiant or luminous flux measurements by use of integrating spheres. The case study in the previous section learns that fluorescence errors can be reduced to a negligible degree by minimizing the phosphor to sphere surface ratio and using low-CCT reference (or auxiliary) sources. Moreover, average integrating sphere reflectance coefficients should not exceed 0.96 for flux measurements of remote phosphor type LED light sources. Still, fluorescence errors largely depend on the phosphor properties of the device under test (cf. Table 1).

If significant fluorescence errors do occur and have to be corrected for, this can be achieved by application of a method that is similar to spectrometer stray light correction by matrix multiplication ${ }^{[10]}$. Performing spectral radiant flux measurements and knowledge of the spectral fluorescence coefficient (e.g. provided by the phosphor manufacturer) are obliged for this approach.

Using matrix notations and algebra, a measured spectral signal $S^{M}$ equals the sum of the true spectrum $S^{0}$ and the fluorescence error on this signal $F \times S^{0}$ so that

$$
S^{0}=I+F^{-1} \times S^{M}
$$


if $I$ represents the unity matrix having the same dimensions as the fluorescence matrix $F=\pi R_{p}\left[\begin{array}{cc}1-\bar{\rho} & \lambda^{\prime}\end{array}\right]^{-1} f_{\lambda} \lambda, \lambda^{\prime}$. Spectra are noted per nanometre. With array $V$ denoting the spectral eye sensitivity function $V \lambda$, the total relative fluorescence error on the luminous flux is then determined as

$$
\varepsilon_{r}=\frac{V \times\left[S^{M}-S^{0}\right]}{V \times S^{0}}=\frac{V \times\left[F \times S^{0}\right]}{V \times S^{0}}
$$

in full agreement with Eq. (15) in the second section. Eqs. (16) and (17) thus provide a fluorescence error correction and evaluation algorithm based on simple matrix algebra. This methodology can be applied in addition to existing LED flux measurement recommendations and standards ${ }^{[15][16]}$.

\section{CONCLUSIONS}

The relative spectral radiant flux error caused by phosphor fluorescence during integrating sphere measurements is first studied theoretically. Two commercially available remote phosphor type LED light sources are used to validate the theory afterwards. Each phosphor's spectral radiance coefficient and spectral fluorescence coefficient is determined with a double monochromator and goniophotometer. Spectral radiant flux measurements of an external blue LED with and without the remote phosphor LED light sources mounted inside the sphere are then compared. A very good agreement between measured and calculated spectral radiant flux errors has been found for both phosphor samples, with deviations smaller than $1 \%$. However, fluorescence contributions are significantly bigger for applications with low CCT, related to high photon absorption coefficients.

Relative fluorescence errors linearly depend on the phosphor to sphere surface ratio, but increase with increasing average sphere reflectance and increasing CCT of a black body reference source. For one of the devices under test, errors obtained at high reflectances and CCTs are of the order of $10 \%$ and therefore unacceptable. Fluorescence contributions can however be reduced to a negligible degree by minimizing the phosphor to sphere surface ratio and using low-CCT reference sources. Average integrating sphere reflectance coefficients should not exceed 0.96. Additionally, a fluorescence error correction and evaluation algorithm based on simple matrix algebra is provided. The methodology can be applied in addition to existing LED flux measurement recommendations and standards.

\section{ACKNOWLEDGEMENTS}

The authors would like to thank the National Institute of Standards and Technology (Gaithersburg, MD, USA) for its financial research support.

\section{REFERENCES}

[1] CIE, "The measurement of luminous flux," Technical Report 084-1989 (1989).

[2] Ohno, Y. and Bergman, R. S., "Detector-referenced sphere photometry for industry," J. IES 32(2), 21-26 (2003).

[3] Miller, C. C. and Ohno, Y., "Luminous flux calibration of LEDs at NIST," Proc. 2nd CIE Expert Symposium on LED Measurement (2001).

[4] Hanselaer, P., Keppens, A., Forment, S., Ryckaert, W. R., and Deconinck, G., “A new integrating sphere design for spectral radiant flux determination of light-emitting diodes,” Meas. Sci. Technol. 20(95111), 1-9 (2009). 
[5] Hoelen, C., Borel, H., de Graaf, J., Keuper, M., Lankhorst, M., Mutter, C., Waumans, L., and Wegh, R., "Remote phosphor LED modules for general illumination - towards $200 \mathrm{~lm} / \mathrm{W}$ general lighting LED light sources," Proc. SPIE 7058, 1-10 (2008).

[6] Ohno, Y., "Integrating sphere simulation: application to total flux scale realization,” Appl. Opt. 33(13), 26372647 (1994).

[7] CIE, “International lighting vocabulary," CIE Publ. no. 17.4 (1987).

[8] Rohwer, L. S. and Martin, J. E., "Measuring the absolute quantum efficiency of luminescent materials,” J. Lumin. 115, 77-90 (2005).

[9] Obein, G., Bousquet, R., and Nadal, M., "New NIST reference goniospectrometer," Proc. SPIE 5880, 1-10 (2005).

[10] Zong, Y., Brown, S. W., Johnson, B.C., Lykke, K. R., and Ohno, Y., "Simple spectral stray-light correction method for array spectroradiometers," Appl. Opt. 45(6), 1111-1119 (2006).

[11] Zhang, Y., Li, L., Zhang, X., and Xi, Q., "Temperature effects on photoluminescence of YAG:Ce3+ phosphor and performance in white light-emitting diodes," J. Rare Earths 26(3), 446-449 (2008).

[12] Keppens, A., Zong, Y., Ohno, Y., Deconinck, G., and Hanselaer, P., 'Determining phosphors' effective quantum efficiency for remote phosphor type LED modules," Proc. CIE Tutorial and Expert Symposium on Spectral and Imaging Methods for Photometry and Radiometry (2010).

[13] Zong, Y. and Ohno, Y., "New practical method for measurement of high-power LEDs," Proc. CIE Expert Symposium on Advances in Photometry and Colorimetry (2008).

[14] Keppens, A., Ryckaert, W. R., Deconinck, G., and Hanselaer, P., "Modeling high-power light-emitting diode spectra and their variation with junction temperature,” J. Appl. Phys. 108(43104), 1-7 (2010).

[15] CIE, "Measurement of LEDs," Technical Report CIE127:2005 (2005).

[16] IES, "Approved Method: Electrical and Photometric Measurements of Solid-State Lighting Products," IES LM79-08, 1-23 (2008). 